\title{
Improving performance of large thrust bearings through modeling and experimentation
}

\author{
L. Dąbrowski, P. Pajączkowski, G. Rotta, M. Wasilczuk and and Wodtke \\ Gdansk University of Technology, Faculty of Mechanical Engineering, Narutowicza 11/12, 80-233 Gdansk, Poland
}

Received 18 April 2013, Accepted 30 May 2013

\begin{abstract}
Large thrust bearings are highly loaded machine elements and their failures cause serious losses. Start ups and stoppages of the bearing under load are specially critical regimes of operation. Load carrying capacity depends on the profile of the oil gap. In transient states this profile is also changing. In the design of large thrust bearings minimizing thermo-elastic deformations is an important goal, which can be accomplished due to application of advanced models of the bearing. Modeling of transient states becomes even more complex since there is a dynamic development of temperature distribution and deformations. Often hydrostatic jacking systems are also used. It seems to the authors that advanced bearing models are applied only in research and development of the bearings while very simple modeling is applied in on-line analysis of data from monitoring systems. Analysis of the measurement data with the use of more sophisticated models may be helpful in assessment of current bearing status - especially in early warning. Material issues create a separate problem for modeling, being more important nowadays as polymer lined bearings come into use. The models used for polymer lined bearings require realistic treatment of heat exchange and resilience of the bearing surface layer.
\end{abstract}

Key words: Thrust bearings / fluid film models / hydrostatic jacking

\section{Introduction}

Thrust bearings are very important components especially in vertical shaft water turbo-generators because they carry weight of the whole machine (several hundred tons in many cases, with sliding speeds reaching $50 \mathrm{~m} . \mathrm{s}^{-1}$ and heat dissipation reaching $1 \mathrm{MW}$ in the largest applications). Thermo-elastic deformations are the main problem in their design and operation, especially critical for large bearings [1]. Another important problem is excessive temperature which is dangerous for bearing alloys. Start ups and stoppages of the bearing loaded with the weight of the rotating parts are especially critical regimes of operation, because of excessive deformations and inadequate film pressure generation at lower rotational speed.

Elements which should be included in a full theoretical model of a thrust bearing are shown in Figure 1. Such a model should include a solution of a set of differential equations for particular regions of a bearing with the use of appropriate boundary conditions (Fig. 1):

I. Fluid film.

II. Bearing pad.

III. Oil bath under the pad.

\footnotetext{
${ }^{a}$ Corresponding author: mwasilcz@pg.gda.pl
}

IV. Collar.

V. Pad supporting mechanism.

VI. Bottom wall of an oil bath.

VII. Zone between the pads.

VIII. Oil inlet to the housing from the cooler.

IX. Oil outlet from the housing.

Modeling of the fluid film bearings has become more and more sophisticated over the years and models evolved from one-dimensional isothermal solutions into threedimensional solutions including many of the elements shown in Figure 1. From contacts with bearing and machinery manufacturers the authors have the impression that sophisticated models are too complex to be used in more routine bearing design.

The present state of modeling is advanced as far as the hydrodynamic film modeling is concerned, but much more backward in modeling of film surroundings, such as lubricant mixing between the pads, deformations of the collar, heat transfer from the collar and pads, geometrical irregularities (chamfers and hydrostatic jacking pockets). Another fact, important in the authors' opinion is a big discrepancy between complexity of models used for bearing development and in condition monitoring. The ones used in condition monitoring are far more basic, or even no model is used to interpret the measurement data. 


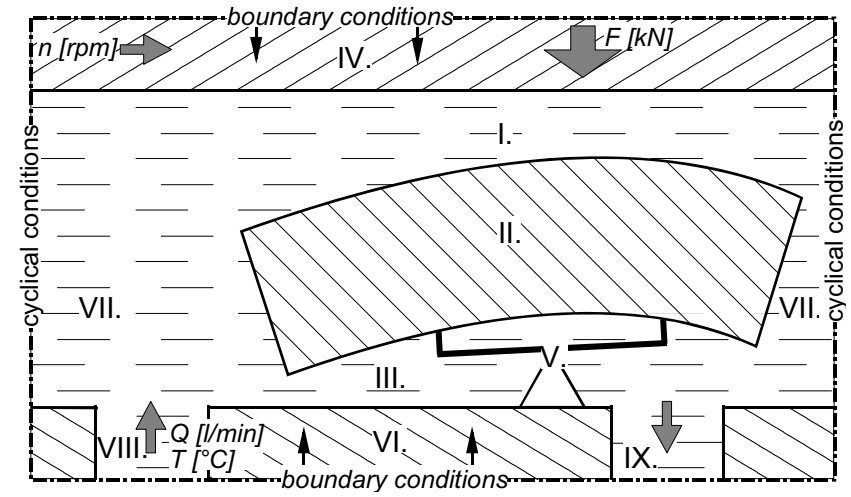

Fig. 1. Elements of a theoretical model of a fluid film thrust bearing.

\section{Problems of design - deformations, temperature, start ups and stops}

Load carrying capacity of a fluid film bearing based on hydrodynamic pressure generation depends on the profile of the oil gap, which is a result of deformations and displacements of bearing elements. In transient states this profile is also changing. In the design of large thrust bearings limiting thermoelastic deformation of the pads is an important goal. Accomplishment of this goal at the design stage is based on application of realistic models of the bearing comprising heat exchange phenomena in the film, between the pads and on the film-structure boundaries, as well as elastic and thermal behavior of the bearing structure. Such models are not common even for application in steady state regimes.

\subsection{Film profile and temperature distribution}

In the literature numerous advanced thrust bearing codes were presented (e.g. [2-4]) however it is usually not possible to make direct comparisons between the results of such programs because many details remain unknown. That was the reason of carrying out a direct comparison of results obtained with the use of different calculation procedures in close cooperation of two research teams, so that all assumptions and details could have been explained. The team at Gdansk University of Technology has devised a thermo-elasto-hydrodynamic (TEHD) model and procedures for evaluation of thrust bearing performance. Main assumptions were described in reference [5]. The comparisons were performed using Fillon's 3D model, in which an advanced TEHD procedure for thrust bearings is impelemented [4]. The results are described in more detail in reference [6]. Main difference of both models is the method of considering variation of lubricant viscosity - in Gdansk University of Technology model viscosity across film thickness is considered constant and calculated for an average film temperature in a given radial and circumferential position - this model is further referred to as $2 \mathrm{D}^{\prime}$ model. In Fillon's model, viscosity variation in all
Table 1. Main bearing parameters and operational conditions of the thrust bearing studied in [5].

\begin{tabular}{lc}
\hline Outer bearing diameter $[\mathrm{mm}]$ & 228.6 \\
Number of pads [-] & 6 \\
Pad angle, [deg] & 50 \\
Rotational speed [rpm] & 3000 \\
Lubricant inlet temperature $\left[{ }^{\circ} \mathrm{C}\right]$ & 50 \\
Lubricant inlet viscosity $[\mathrm{Pa} . \mathrm{s}]$ & 0.03819 \\
\hline
\end{tabular}

directions is considered (this model will be further described as 3D). One of the cases for which both models were compared was the bearing described and studied in references $[5,7]$. Its basic parameters are given in Table 1 .

Calculation results are compared in Figure 2. One may see two different results for the $2 \mathrm{D}^{\prime}$ model - one is obtained with the same inlet temperature as in 3D model, while in the other calculation a different inlet temperature was used. This different inlet temperature was calculated with the use of Ettles method [8]. According to this method "hot oil carry over factor" depending on the sliding speed in the bearing and the distance between bearing pads is fixed with the use of a special graph and then used to evaluate inlet temperature as a function of outlet and supply temperatures. Modification of the value of boundary condition caused that the results of minimum film and maximum temperature in $3 \mathrm{D}$ and $2 \mathrm{D}^{\prime}$ models do not differ substantially. However, a great difference is observed for friction losses calculations.

The selected results (maximum temperature and minimum film thickness values), may seem quite similar in 3D and $2 \mathrm{D}^{\prime}$ with modified boundary condition, but a closer look at the results shows that the distribution of the temperature and film profile differ much (Tab. 2). Point of maximum temperature is shifted towards outer radius and the isotherms differ in shape. Different temperature distribution at the surface changes thermal deformations of the pad and, in consequence, the film profile is altered. It can also be noticed that in each of the models the angle of pad tilt (assessed as the difference between maximum and minimum film thickness) is different. The shape of the pad sliding surface also differs in various models the models. In the $2 \mathrm{D}^{\prime}$ model with higher inlet temperature convexity of the pad is larger, which can be judged on the basis of film thickness contours shape. Due to more favourable (e.g. less convex) shape of the sliding surface a given load can be carried with larger minimum oil film thickness in the bearing and with its improved reliability.

\subsection{Realistic boundary conditions}

In all models setting boundary conditions becomes an important issue. Boundary conditions for a fluid film bearing depend on the scope comprised by the model. If the model comprises fluid film only then conditions of on the fluid solid interfaces and film inlet/outlet/periphery are the boundary conditions. In this case the model itself is relatively simple but the boundary condition values are difficult to assume and verify since the boundaries are 

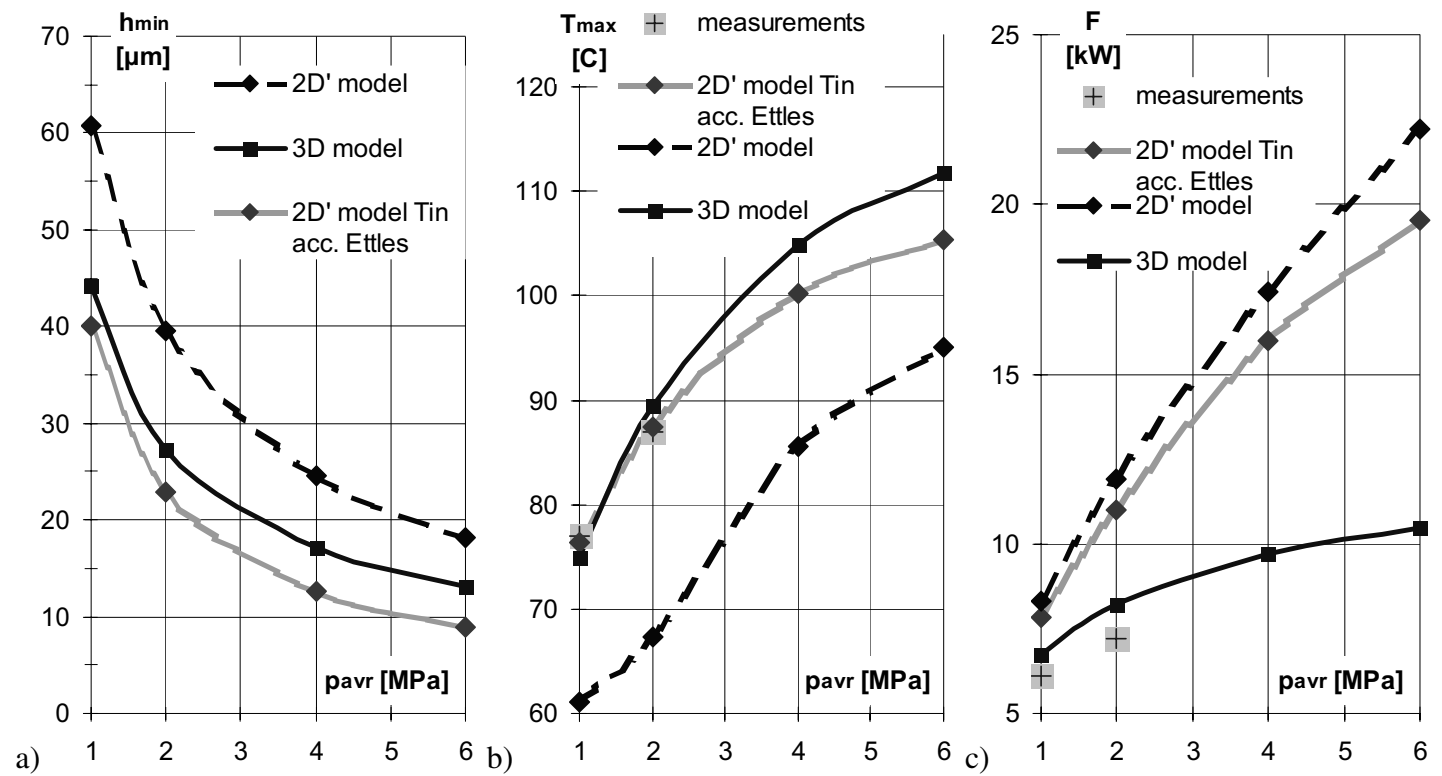

Fig. 2. Results of TEHD calculations with the use of $2 \mathrm{D}^{\prime}$ and $3 \mathrm{D}$; (a) minimum film thickness $h_{\min }[\mu \mathrm{m}]$, (b) maximum temperature $T_{\max }\left[{ }^{\circ} \mathrm{C}\right]$, (c) friction losses $F[\mathrm{~kW}]$.

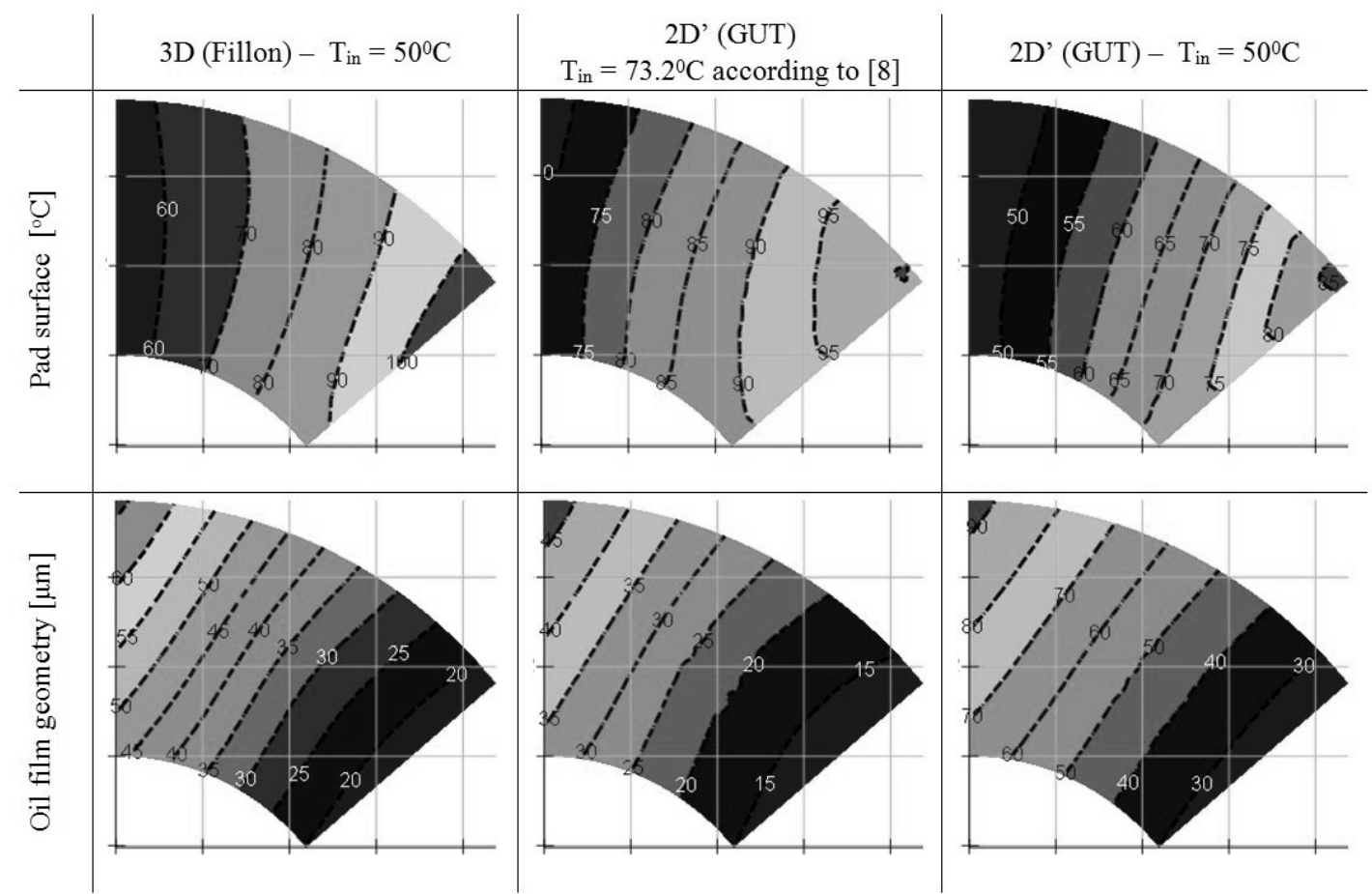

Table 2. Comparison of surface temperature distribution and film profile calculated with the use of $3 \mathrm{D}, 2 \mathrm{D}^{\prime}$ and $2 \mathrm{D}^{\prime}$ with increased inlet temperature, $p_{\text {avr }}=4[\mathrm{MPa}]$.

not easily accessible for experiments. On the other hand, if one is able to build more complex models comprising more elements and including more phenomena then some of the previously mentioned boundaries become integral part of the model. The boundaries are then shifted outwards and boundary conditions become more accessible for experimental verification. Such more distant boundary conditions will probably have less pronounced influ- ence on the phenomena in the film, which is the most important for bearing performance. A simple example of problems is the case of setting boundary conditions at film inlet - these would be an assumed temperature profile (or more usually one constant temperature) and velocity profile.

The influence of inlet temperature can be shown in Figure $3[9]$, in which the results of two sets of a thrust 

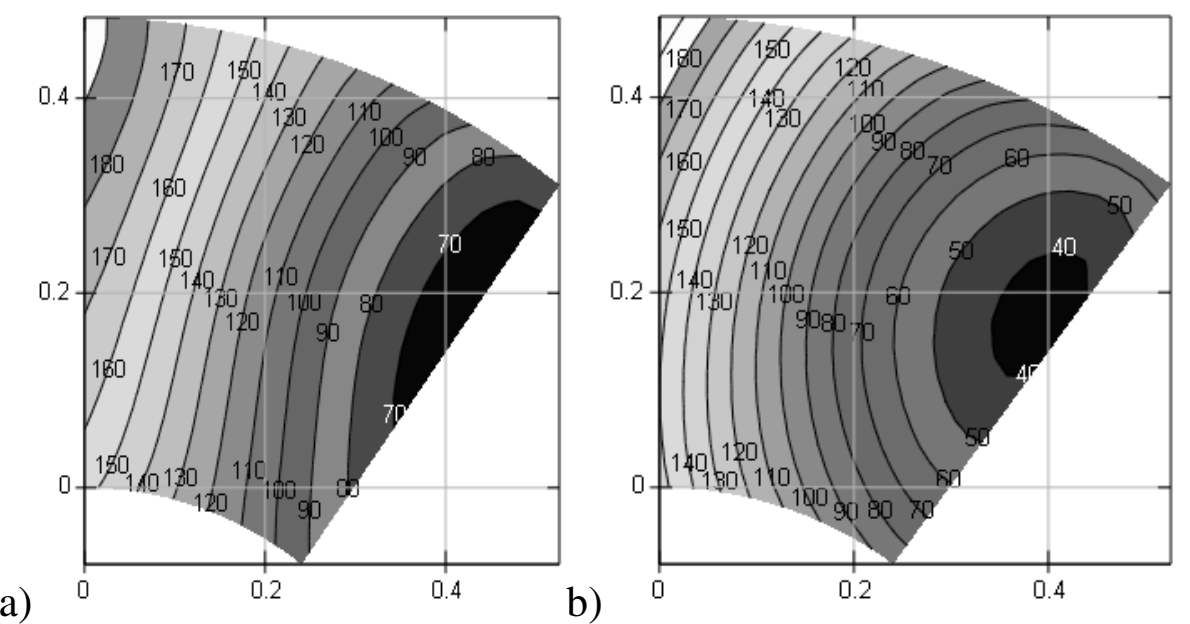

Fig. 3. Film profile for inlet temperature of (a) $40{ }^{\circ} \mathrm{C}$, (b) $55{ }^{\circ} \mathrm{C}[9]$.
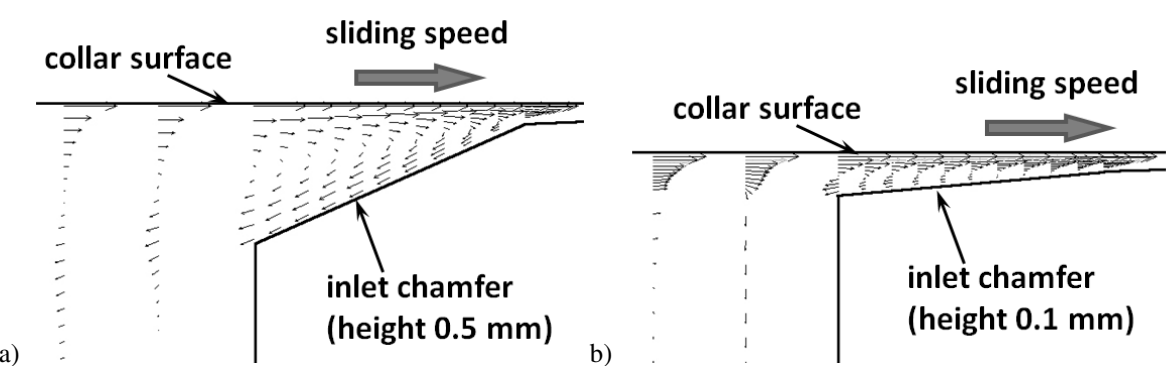

Fig. 4. Velocity distribution in an inlet zone of a thrust bearing pad: (a) chamfer height $0.5 \mathrm{~mm}$, (b) chamfer height $0.1 \mathrm{~mm}$.

bearing calculations are compared. The only difference is inlet temperature, being $40{ }^{\circ} \mathrm{C}$ in one case and $55^{\circ} \mathrm{C}$ in the other. The results show large differences between the two cases with different film profiles (shown in Fig. 3) and also temperature distribution, with maximum temperatures, respectively equal to $64.8^{\circ} \mathrm{C}$ and $71.7^{\circ} \mathrm{C}$. Maximum film pressure calculated for both inlet temperatures also differ considerably by almost $2 \mathrm{MPa}$.

More detailed study of the film inlet was carried out with the use of CFD (Computational Fluid Dynamics) $[10]$. The results showed that the temperature in the inlet to the film was not constant and that the velocity profile differed much depending on details of bearing design. The influence of inlet chamfer, detail which is usually omitted in fluid film models, on inlet velocity profile is shown in Figure 4. In the analysed case, maximum bearing temperature differs by $3{ }^{\circ} \mathrm{C}$ between the bearing with $0.1 \mathrm{~mm}$ chamfer and $0.5 \mathrm{~mm}$ chamfer. This difference is most probably caused by more efficient flow of fresh cool oil into the oil gap in the case of higher chamfer, while in the case of chamfer height equal to $0.1 \mathrm{~mm}$ the amount of hot oil entering the film is larger.

Similar difficulties and doubts arise when one tries to set values of convection coefficients at the back of the pad. In this case, the design of pad support and housing have a great influence on intensity of oil circulation and, as a result, on convection coefficient. In some designs the pad supporting disc is large and covers almost whole surface of the pad back affecting heat convection, while in the others (e.g. spring mattress support) oil is free to move under the pad.

\subsection{Behavior in the transient states}

According to many authors deformations are important for performance of large thrust bearings. Calculations and field data show that deformation in transient states may be larger than in steady state because of low temperature of the oil bath and increased thermal gradients (Fig. 5) - that is why analysis of transient states is of great importance.

Modeling of transient states is complex since there is a dynamic development of temperature distribution and deformations. Additionally in most of the cases, hydrostatic jacking systems are used allowing hydrodynamic lubrication to be changed into a hybrid mode of lubrication. The authors' attempts to develop thrust bearing model suitable for analysis of transient states and including hydrostatic/hybrid mode of operation are summarized in a separate paper [13]. The devised model utilizes the technique of fluid structure interaction (FSI) realized by combination of finite element method (FEM) and computational fluid dynamics (CFD). This model was used to study the reasons of bearing failure in one of the power plants - the 

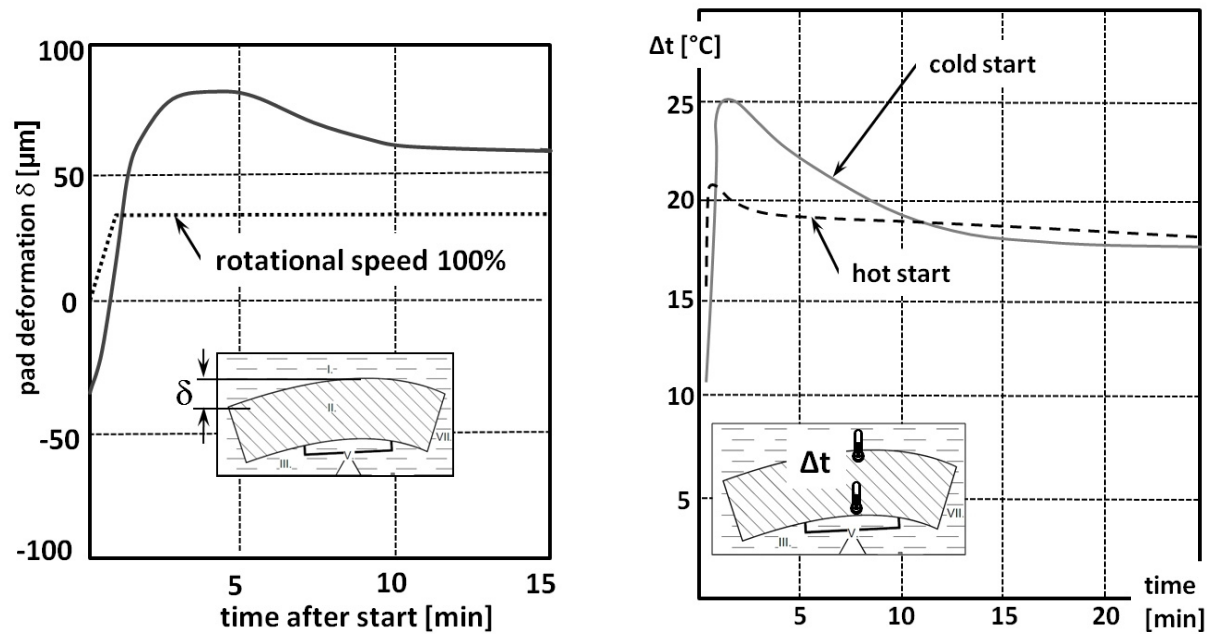

Fig. 5. Measured deformation [11] and temperature difference [12] in large thrust bearing pad.
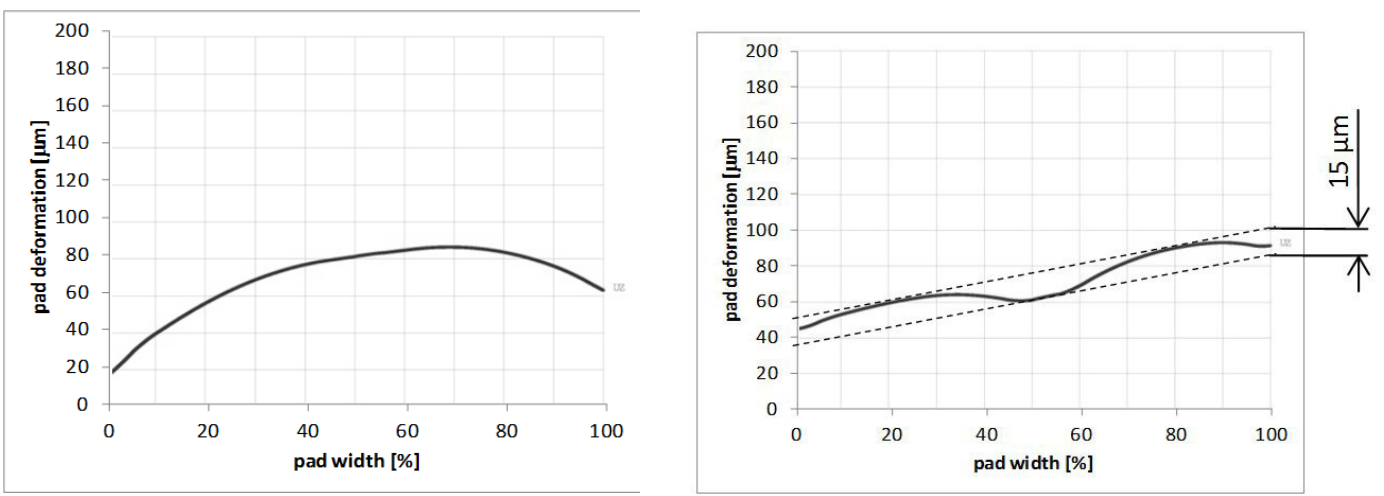

Fig. 6. Pad surface radial profiles: in steady state (left) and transient state (right).

results show great difference in radial and circumferential pad profiles between steady state and in transient state with low oil bath temperature (Figs. 6 and 7). Concavity of the pad surface was caused by injection of cold oil into the hydrostatic pocket. Load carrying capacity with such a pad profile was too low to prevent bearing seizure in the case of hydrostatic system failure. Other analysis, as well as measurements at the commissioning, showed that the bearing was able to stop safely without activation of a hydrostatic jacking system only if oil bath temperature was higher than $25^{\circ} \mathrm{C}$.

\subsection{Material issues - polymer lined bearings}

Material issues in polymer lined bearing create a separate problem for modeling and experimentation. The resilience, heat conductivity and other features of polymer surface layers differ much from these for metallic bearing alloys [14]. Different material properties yield differences in results of calculations of temperature distribution and deformed shape of the bearing surface. These properties have been included in the analyses presented in $[7,15]$. Use of reference values for unfilled PTFE or PEEK may be justified in some cases but the bearing manufacturers tend to use polymers filled with various fillers to improve tribological properties. The fillers have a great influence on elastic and thermal properties of polymers. The authors of this paper are not aware of availability of material data for particular compositions of polymers being used in currently manufactured and used polymer lined bearings. The other difficulty lies in composite character of the polymer surface layers - in various bonding techniques part of the polymer layer is reinforced with metalwire mesh (Fig. 8a) or porous bronze (Fig. 8b) [14]. Complex structure of this composite layer has an influence on both - elastic and thermal properties of the lining. Because of complexity and irregularity of a composite layer, its thermal and elastic properties have to be experimentally measured in order to obtain relevant input data for bearing models.

\section{On-line analysis of bearing performance}

The most important operational parameters of any fluid film thrust bearing are (minimum) film thickness and (maximum) temperature. These parameters are very 

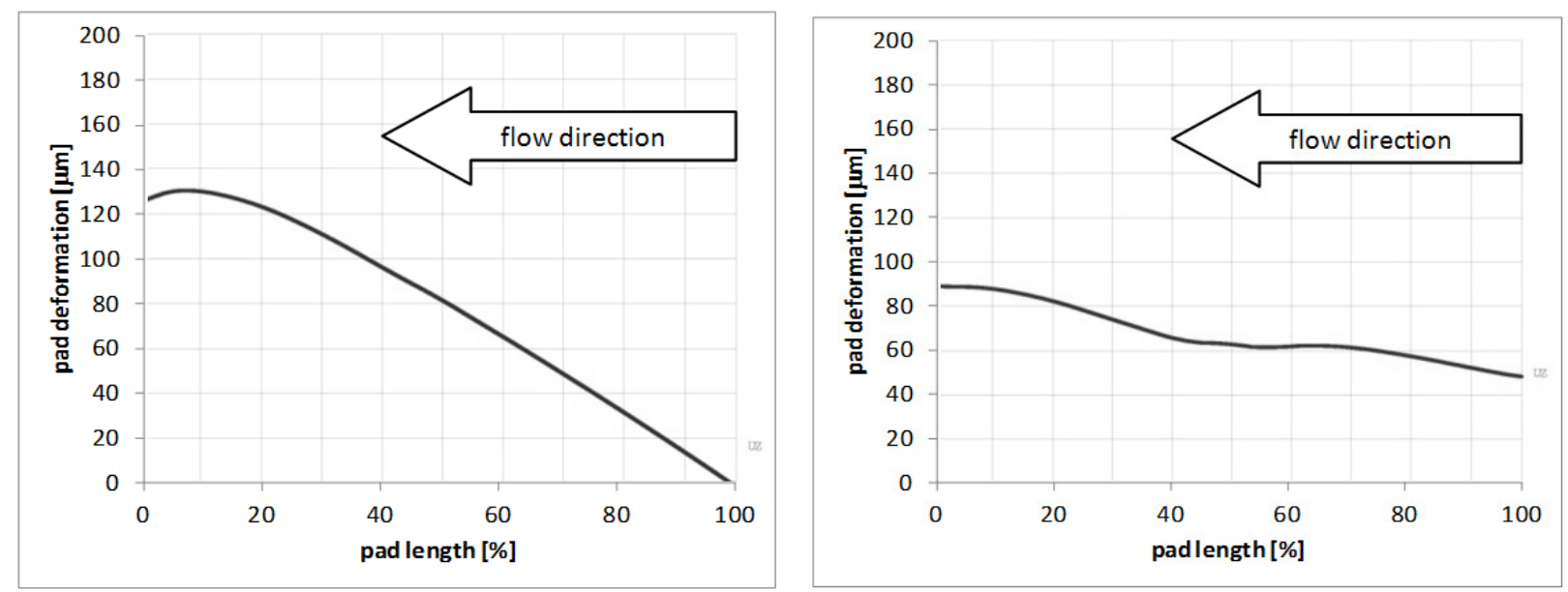

Fig. 7. Pad surface tangential profiles: steady state (left) and transient state (right).
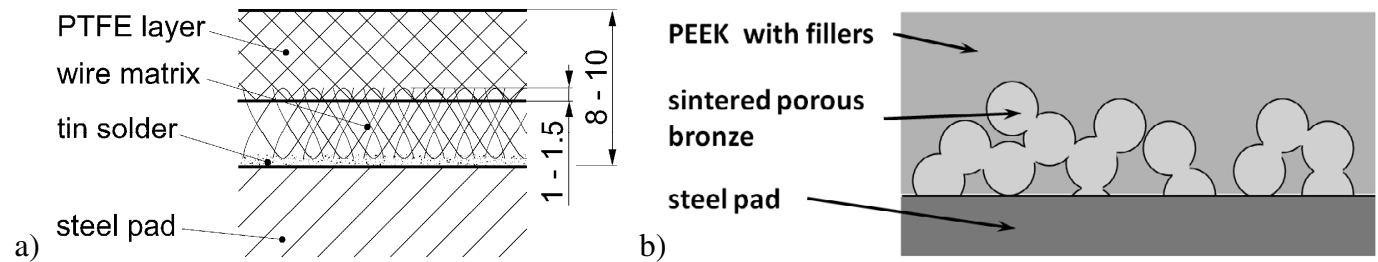

Fig. 8. Irregular structure of polymer lining of thrust bearing pads (a) PTFEwire mesh composite (b) PEEKporous bronze composite.

difficult to be directly measured just because of difficult access to the film. Seemingly direct bearing temperature measured with the sensors mounted in a bearing pad under the surface is not the source of direct information on temperature in hot spots of the bearing surface or film because the hottest temperature may not be directly over the sensor. Additionally, there is also some temperature gradient between the surface and the sensor (large difference occurs especially in polymer lined bearings). The other example is film measurement with the use of sensors mounted at the pad side (Fig. 9). With the use of such a sensor one can only measure film thickness at the sensor position ("measured distance from the collar") while the real minimum value ("real minimum film thickness") differs much because of many disturbing factors - pad deformations (" $\delta$ ") being the most substantial (Fig. 10).

The above examples illustrate the need for the use of inverse methods in which with the use of measurements and modeling one may evaluate the direct value of the crucial parameter. If, in addition, such models can be simple allowing for fast calculations the evaluation of the parameters may be performed in real time during bearing operation. Such calculations would be a powerful aid for bearings condition monitoring. A diagnostic model should consider elements shown in Figure 1, but the functions should comply with the values measured in the monitoring system instead of fulfilling boundary conditions (Fig. 11). From the point of view of calculation methods, such formulation of a problem is the so called "inverse

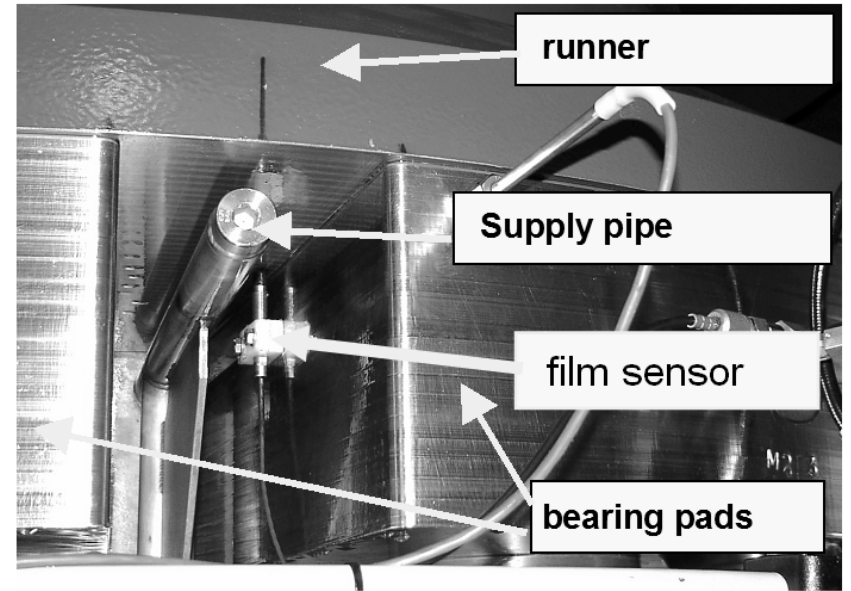

Fig. 9. Thrust bearing with film thickness sensor mounted next to the pad wall [9].

problem". Inverse problems have become more utilized in recent years. Methodology of solving inverse problems is being developed (e.g. [16,17]). However existing solutions can not be directly used for solving an inverse problem of fluid film bearings, because of small number of temperature measurement points and large temperature gradients. The other problem is the effect of measurement errors on accuracy of calculations.

An example of such an inverse model used for analysis of bearing measurement data is an idea of a 


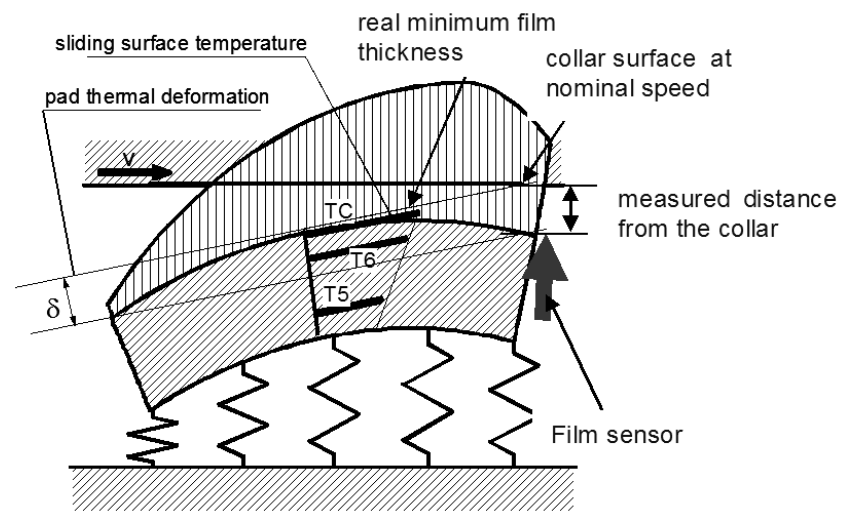

Fig. 10. Difference between measured distance from the collar and minimum film thickness.

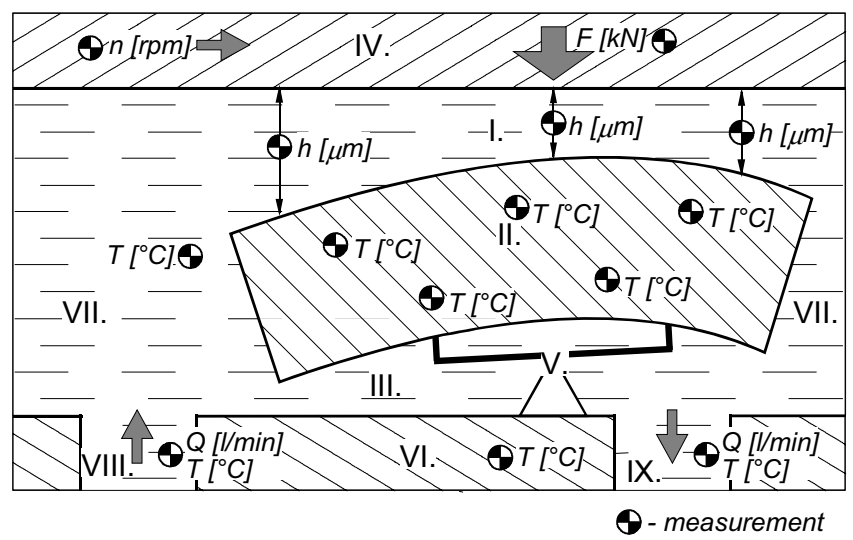

Fig. 11. Elements of a diagnostic model of a fluid film thrust bearing.

diagnostic model based on Inverse Heat Conduction Problem (IHCP) proposed in reference [18]. The distribution of the heat flux entering the bearing pad can be used for decisions taken up either by a machine operator or even by an automated expert system. Heat flux is the quantity that illustrates the friction phenomena in the bearing: for example in mixed lubrication regime the amount of heat generated increases by the orders of magnitude. Decisions in the process of controlling machine operation can be taken on the basis of:

1. maximum value of the heat flux - it is possible to evaluate in experiments the allowable heat flux that would not cause babbit destruction;

2. time after which surface temperature would have reached an ultimate (destruction) temperature - such calculations will probably be possible on-line with the assumption of constant heat flux.

Temperature field and the quantity of heat entering the pad are evaluated by the techniques devised with the use of methods of IHCP and it is supposed that on-line calculations will be possible so that the changes can be monitored on-line. Heat flux increase can be relatively quickly evaluated and observed. What is more important is that this value is independent from ambient temperature, cooling water temperature, etc. Also in case of some failures during transient states, excessive heat flux will be observed and will provide information for emergency shutdown even though the bearing temperature will be lower than emergency shutdown level of temperature.

\section{Conclusions}

The authors tried to show some aspects of use of modeling for improved accuracy of predicting bearing performance. Improved modeling seems necessary to study transient states of bearings. It is commonly accepted that improved models can be a tool for bearing development and the classical calculation models of thrust bearings are quite well developed. They are nowadays sophisticated and take into account a number of important phenomena. On the other hand in condition monitoring systems of thrust bearings, very primitive models are used (if any) despite great consequences of bearing failures. Operation is based on direct readings of measured parameters and comparing them to threshold values, although these measurements are not precise and do not give direct results of parameters crucial for bearing safe operation. It seems to the authors that bearings reliability may be substantially increased by the use of specially devised inverse models, which could be used for advanced online analysis of data from condition monitoring systems.

Acknowledgements. Parts of the presented research were carried out within the scope of research projects No. 4 T07C 017 29 and No. 4 T07C 00630 funded by Polish Ministry of Science and Higher Education

\section{References}

[1] C.M. Ettles, Size effects in tilting pad thrust bearings, Wear 59 (1980) 231-245

[2] C.M. Ettles, Three dimensional computation of thrust bearings, Proceedings of the 13th Leeds Lyon Symposium on Tribology, Elsevier, 1987, pp. 95-104

[3] K. Kim, M. Tanaka, Y. Hori, A Three-Dimensional Analysis of Thermohydrodynamic Performance of SectorShaped, Tilting-Pad Thrust Bearings, Trans. ASME, J. Lubr. Technol. 105 (1983) 406-413

[4] S.B. Glavatskih, M. Fillon, TEHD Analysis of Thrust Bearings With PTFE-Faced Pads, J. Tribol. 128 (2006) $49-58$

[5] L. Dąbrowski, M. Wasilczuk, Evaluation of a water turbine hydrodynamic thrust bearing performance on the basis of thermoelastohydrodynamic calculations and operational data, Proceedings of the IME, Vol. 218 Part J: Journal of Engineering Tribology 218 (2004) 413-421

[6] M. Wodtke, M. Wasilczuk, M. Fillon, Predicting performance of thrust bearings with use of contemporary models, Proceedings of the 7th EdF/LMS Poitiers Workshop, Futuroscope 2008, Paper P, Poster session

[7] S. Glavatskich, M. Fillon, R. Larsson, The Significance of Oil Thermal Properties on the Performance of a TiltingPad Thrust Bearing, Trans. ASME, J. Tribol. 124 (2002) 377-385 
[8] C.M. Ettles, Hot Oil Carry Over in Thrust Bearings, Proc. IME, Part 3L 184 (1970) 75-81

[9] M. Wasilczuk, G. Rotta, Modeling lubricant flow between thrust-bearing pads, Tribol. Int. 41 (2008) 908-913

[10] M. Wasilczuk, G. Rotta, CFD Analysis of the Lubricant Flow in the Supply Groove of a Hydrodynamic Thrust Bearing Pad, Proceedings of the STLE/ASME International Joint Tribology Conference IJTC2007, Paper no IJTC2007-44304

[11] K. Kawaike, K. Okano, Y. Furukawa, Performance of a Large Thrust Bearing with Minimized Thermal Distortion, ASLE Trans. 22 (1977) 125-134

[12] C.M. Ettles, J. Seyler, M. Bottenschein, Some Effects of Start-Up and Shut-Down on Thrust Bearing Assemblies in Hydro-Generators, Trans. ASME, J. Tribol. 125 (2003) 824-832

[13] P. Pajączkowski, M. Wasilczuk, A. Schubert, Modeling Transient States of Large Hydrodynamic Thrust Bearings, Proceedings of the 7th EdF/LMS Poitiers Workshop, Futuroscope 2008, Paper O, Poster session
[14] S. Glavatskih, M. Wasilczuk, M. Fillon, Performance peculiarities of PTFE-faced tilting-pad thrust bearings, Proceedings of the 3rd EdF/LMS Poitiers Workshop. Futuroscope 2004, Paper K.

[15] C.M. Ettles, R.T. Knox, J.H. Ferguson, D. Horner, Test Results for PTFE-Faced Thrust Pads, With Direct Comparison Against Babbitt-Faced Pads and Correlation With Analysis, J. Tribol. 125 (2003) 814-823

[16] J. Zueco, F. Alhama, C.F. Gonzalez-Fernandez, Numerical nonlinear inverse problem of determining wall heat flux, Heat Mass Transfer 41 (2005) 411-418

[17] H.M. Park, S.W. Jung, On the solution of multidimensional inverse heat conduction problem using an efficient sequential method, ASME J. Heat Transfer 123 (2001) $1021-1029$

[18] L. Dąbrowski, Temperature measurements in monitoring of large thrust bearings, Proceedings of the 5th EdF/LMS Poitiers Workshop, Futuroscope 2006, Paper F. 